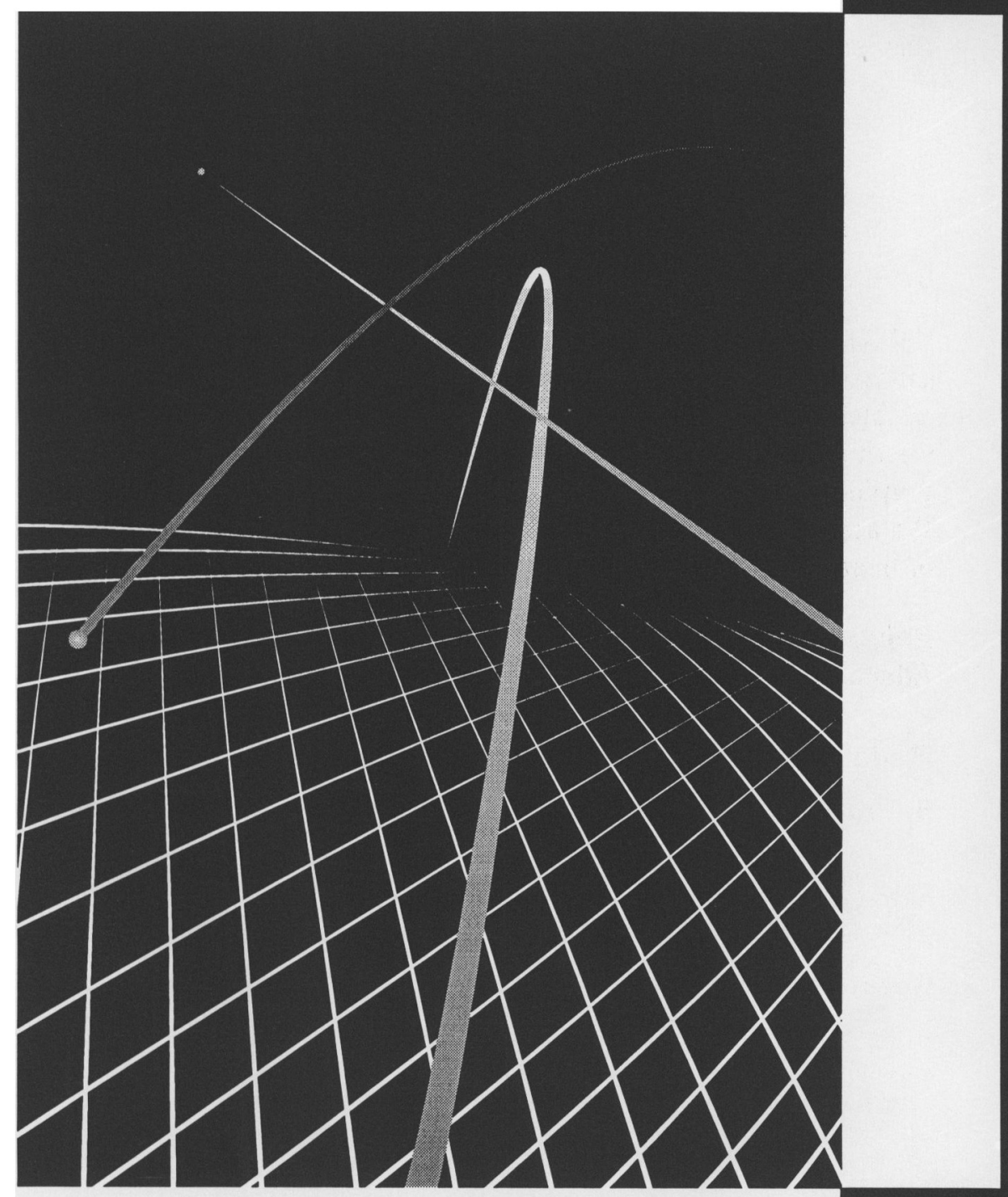

T H E 


\title{
ON THE PROBLEM OF POST-NEWTONIAN
}

\section{ROTATIONAL MOTION}

\author{
SERGEI A. KLIONER ${ }^{1}$ \\ Lohrmann Observatorium, Institut für Planetare Geodäsie \\ Dresden, Germany
}

\begin{abstract}
The problems of modeling of the rotational motion of the Earth are considered in the framework of general relativity. Both, rigid and deformable bodies are discussed. Rigorous definitions of the tensor of inertia, Tisserand-like axes and the angular velocity of rotation of an extended deformable body moving and rotating in external gravitational fields are proposed in the first post-Newtonian approximation. The implications of these post-Newtonian definitions on modeling of Earth rotation are analyzed.
\end{abstract}

\section{Introduction}

Rotational motion of extended bodies in general relativity is a complicated problem which has no complete solution up to now. On the other hand the observational accuracy of modern geodynamical observations is gradually approaching a level of 10 microarcsecond which forces us to think of theoretical models of Earth rotation having an accuracy of 1 microarcsecond. This makes it indispensable to have a rigorous description of Earth rotation in the framework of general relativity.

The concept of spatial rotation is intimately related to the symmetries of 3-dimensional Euclidean space of Newtonian mechanics and cannot be generalized in a physically meaningful way onto general relativity which deals with curved 4-dimensional space-time. Although in some special cases the space-time of general relativity does have an axis of symmetry and spatial rotation could be rigorously defined, this is certainly not the case when we consider the translational and rotational motion of the Earth in the gravitational field of other bodies of the Solar system.

It is well known that a description of any observable physical process in general relativity must be coordinate-independent to be physically mea-

${ }^{1}$ On leave from the Institute of Applied Astronomy, St. Petersburg, Russia

I. M. Wytrzyszczak, J. H. Lieske and R. A. Feldman (eds.),

Dynamics and Astrometry of Natural and Artificial Celestial Bodies, 383, 1997.

(C) 1997 Kluwer Academic Publishers. Printed in the Netherlands. 
ningful. However, modern methods of monitoring Earth rotation are based on determination of positions of Earth-based observing sites with respect to some reference system and subsequent investigation of the time-dependence of the positions. Therefore, relativistic modeling of Earth dynamics should necessarily refer to a relativistic reference system, and we do not have to elaborate a coordinate-independent approach to describe Earth rotation. On the contrary, it is quite sufficient to agree about what we call Earth rotation in the reference system which we use to describe the coordinates of the observing sites. In that case, we can say that the Earth orientation parameters, which describe Earth rotation, are defined operationally (that is by the procedure of measurements) and are aimed at an ad hoc description of Earth rotation only in that particular reference system.

\section{Post-Newtonian Rotational Equations of Motion}

The starting point for a discussion of Earth rotation in both general relativity and Newtonian mechanics is the rotational equations of motion relating the angular momentum of the body and the torque. Rotational equations of motion of extended bodies in the post-Newtonian approximation of general relativity have been investigated by many authors (see Fock, 1959; Brumberg, 1972; Damour, Soffel and Xu, 1993; Klioner, 1996). Provided that a physically adequate geocentric reference $\left(t, x^{i}\right)$ is used, the post-Newtonian rotational equations of motion of the Earth can be represented as (see Brumberg and Kopeikin, 1989; Damour, Soffel and Xu, 1993; Klioner, 1996 for a definition of the reference system and further technical details)

$$
\frac{d}{d t} S^{i}=F^{i}+\mathcal{O}\left(c^{-4}\right)
$$

where $S^{i}$ is the post-Newtonian spin defined as

$$
\begin{gathered}
S^{i}=\varepsilon_{i j k} \int_{V} x^{j} Q^{k} d x^{3}+\mathcal{O}\left(c^{-4}\right) \\
Q^{k}=\sigma^{k}(t, \mathbf{x})\left(1+\frac{4 G}{c^{2}} \int_{V} \sigma\left(t, \mathbf{x}^{\prime}\right) \frac{1}{\left|\mathbf{x}-\mathbf{x}^{\prime}\right|} d x^{\prime 3}\right) \\
-\frac{1}{2 c^{2}} G \sigma(t, \mathbf{x}) \int_{V} \sigma^{s}\left(t, \mathbf{x}^{\prime}\right) \frac{7 \delta^{k s}+n^{k} n^{s}}{\left|\mathbf{x}-\mathbf{x}^{\prime}\right|} d x^{3}+\mathcal{O}\left(c^{-4}\right) \\
n^{i}=\frac{x^{i}-x^{\prime i}}{\left|\mathbf{x}-\mathbf{x}^{\prime}\right|} \\
\sigma=\frac{1}{c^{2}} T^{\alpha \alpha}, \quad \sigma^{i}=\frac{1}{c} T^{0 i}
\end{gathered}
$$


Here $T^{\alpha \beta}$ is the energy-momentum tensor of the matter, and $V$ is the 3 volume covering the Earth. The right-hand side of (1) represents the postNewtonian torque

$$
F^{i}=\varepsilon_{i j k} \int_{V} \sigma(t, \mathbf{x}) x^{j} f^{k} d x^{3}+\mathcal{O}\left(c^{-4}\right)
$$

where $f^{k}$ is a post-Newtonian external tidal force (see Damour, Soffel and $\mathrm{Xu}, 1993$; Klioner, 1996). In analogy to Newtonian physics the postNewtonian torque (6) vanishes for isolated bodies. Let us note that the post-Newtonian rotational equations of motion (1) are not unique. Some terms can be transferred from the right-hand side of (1) into the left-hand side leading to another definition of the post-Newtonian spin $S^{i}$ and the torque $F^{i}$. The representation (1)-(6) has the advantage that the spin $S^{i}$ is explicitly proportional to the time-space components $T^{0 i}$ of the energymomentum tensor which plays an important role below.

\section{Angular Velocity and Tensor of Inertia}

Standard way to split the spin into an angular velocity and a tensor of inertia in Newtonian mechanics is to consider a rigid body. A rigid body is usually defined as a system consisting of a number of point masses which do not move with respect to each other. For a rigid body we can introduce a rigidly rotating reference system in which all the particles of the body are at rest. The angular velocity of the rotating reference system relative to the inertial one is called angular velocity of rotation of the body. The angular velocity and the spin (both of which are uniquely defined) define uniquely a tensor of inertia.

In general relativity it is not trivial to define a rigid body in a physically meaningful way (see Dixon, 1979 for a review). It is completely meaningless to say that the coordinate distances between particles of the body should be constant in some reference system, since it is always possible to consider another reference system, in which the distances would depend on time, and all reference systems covering the space-time region under consideration are equivalent. However, we can define the body to be rigid if physical, coordinate-independent distances between all pairs of the particles are constant. This is the so called Born rigidity, which is sometimes called kinematical rigidity since it is based only on the internal velocity distribution within the body and ignores stresses and energy fluxes contributing to $T^{\alpha \beta}$ (Dixon, 1979). Another approach, which could be called dynamical rigidity, consists in constraining the energy-momentum tensor of the body $T^{\alpha \beta}$ and/or gravitational field produced by the body to satisfy some specific conditions (Dixon, 1979; Thorne and Gürsel, 1983). Thus, Thorne and Gürsel (1983) (see also Soffel, 1994) constructed a mathemati- 
cally self-consistent rigid body model by constraining $T^{\alpha \beta}$ to satisfy

$$
\sigma^{i}=\frac{1}{c} T^{0 i}=\frac{1}{c^{2}} T^{00} \varepsilon_{i j k} \omega^{j}(t) x^{k}+\frac{1}{c^{2}} T^{i s} \varepsilon_{s j k} \omega^{j}(t) x^{k}+\mathcal{O}\left(\omega^{2}\right)+\mathcal{O}\left(c^{-4}\right),
$$

where $\omega^{i}(t)$ can be considered as angular velocity of rotation of the body. According to (2)-(3) this condition makes the post-Newtonian spin $S^{i}$ proportional to the angular velocity $\omega^{i}$ and defines the relativistic tensor of inertia (see Thorne and Gürsel, 1983; Soffel, 1994).

Rigorously speaking a rigid body in general relativity can never change its angular velocity, since this would violate the basic postulate of relativity stating that no physical interaction can propagate at infinite velocity. However, for practical purposes it is much more interesting to consider precessing rigid bodies. For this purpose Thorne and Gürsel (1983) have suggested to neglect everywhere the terms $\mathcal{O}\left(\omega^{2}\right)$ of the second order with respect to angular velocity of the body. There are two kinds of such terms: the terms of order of $\varepsilon_{1}=\omega^{2} L^{2} / c^{2}$ and those of order of $\varepsilon_{2}=\omega^{2} L / g=\varepsilon_{1} \cdot L / m$, where $\mathrm{L}$ is the typical linear size of the body, $g$ is the gravitational acceleration on the surface of the body and $m=G M / c^{2}$ is the gravitational radius of the body. For pulsars with a period of order of 1 second (for which the approach was initially invented) the small parameter $\varepsilon=\max \left(\varepsilon_{1}, \varepsilon_{2}\right) \sim 10^{-7}$. However, for the Earth $\varepsilon \sim 4 \cdot 10^{-3}$ which means that the approach allows to calculate at most 2 digits of the relativistic effects in the rotational motion. Moreover, for Jupiter $\varepsilon \sim 0.1$ and for a typical millisecond pulsar $\varepsilon \sim 0.2$ which makes the whole approach (at least in its present form) inapplicable to those objects.

Although the concept of a rigid body is very attractive, real celestial bodies including the Earth deviate significantly from rigidity. The principle idea to deal with deformable bodies in general relativity is to treat relativistic effects in internal motion within the body as additional deformations and to treat the deformable body in basically the same way as we do in Newtonian mechanics: introduce a rigidly rotating reference system in which the body appears to be at rest on an average in one sense or another and then ascribe the angular velocity of rotation of that reference system to the body itself (see Dixon, 1979; Voinov, 1988; Klioner, 1996). This procedure is quite analogous to the definitions of Tisserand axes and principal axes of inertia of a deformable body in Newtonian mechanics.

Representing formally the time-space component of the energy-momentum tensor as

$$
\sigma^{i}=\frac{1}{c} T^{0 i}=\bar{\sigma}^{i}+\frac{1}{c^{2}} T^{00} \varepsilon_{i j k} \omega^{j}(t) x^{k}+\frac{1}{c^{2}} p^{i s} \varepsilon_{s j k} \omega^{j}(t) x^{k}+\mathcal{O}\left(c^{-4}\right)
$$

(where $\omega(t)$ is an arbitrary function of time, and $p^{i j}$ is the Newtonian stress tensor of the matter) we get

$$
S^{i}=\bar{S}^{i}+C^{i j} \omega^{j}+\mathcal{O}\left(c^{-4}\right)
$$




$$
\begin{aligned}
C^{i j}(t)= & \int_{V} \rho\left(1+\frac{4 U}{c^{2}}\right)\left(\delta^{i j} x^{s} x^{s}-x^{i} x^{j}\right) d x^{3}+\frac{1}{c^{2}} \varepsilon_{i a k} \varepsilon_{j b s} \int_{V} x^{a} x^{b} p^{k s} d x^{3} \\
& -\frac{1}{c^{2}} \frac{G}{2 c^{2}}\left(7 \delta^{i j} \alpha^{s s}-7 \alpha^{i j}+\beta^{i j}\right), \quad \rho=\frac{1}{c^{2}} T^{00} \\
\alpha^{i j}(t)= & \int_{V} \int_{V} \rho(t, \mathbf{x}) \rho\left(t, \mathbf{x}^{\prime}\right) \frac{x^{i} x^{\prime j}}{\left|\mathbf{x}-\mathbf{x}^{\prime}\right|} d x^{\prime 3} d x^{3} \\
\beta^{i j}(t)= & \int_{V} \int_{V} \rho(t, \mathbf{x}) \rho\left(t, \mathbf{x}^{\prime}\right) \frac{\left(\mathbf{x} \times \mathbf{x}^{\prime}\right)^{i}\left(\mathbf{x} \times \mathbf{x}^{\prime}\right)^{j}}{\left|\mathbf{x}-\mathbf{x}^{\prime}\right|^{3}} d x^{33} d x^{3}
\end{aligned}
$$

where $U$ is the Newtonian gravitational potential and $\bar{S}^{i}$ is defined by (2)(3) where $\bar{\sigma}^{i}$ is formally substituted for $\sigma^{i}$. The representation (8) has been chosen such that $C^{i j}$ for a deformable body coincide with the tensor of inertia for a rigid body in the sense of Thorne and Gürsel (see Soffel, 1994) up to terms $\mathcal{O}\left(\omega^{2}\right)$. Some other advantages of (8) are noted in Klioner (1996). Possible definitions of $\omega(t)$ are

1) Post-Newtonian Tisserand axes:

$$
\bar{S}^{i}(t) \equiv 0
$$

2) Post-Newtonian principal axes of inertia:

$$
C^{i j}(t)=P^{i a}(t) P^{j b}(t) \bar{C}^{a b}(t), \quad \bar{C}^{a b}(t)=\left(\begin{array}{ccc}
\mathcal{A}(t) & 0 & 0 \\
\mathbf{0} & \mathcal{B}(t) & 0 \\
\mathbf{0} & 0 & \mathcal{C}(t)
\end{array}\right)
$$

where orthogonal matrix $P^{i j}(t)$ is defined by

$$
\omega^{i}=\frac{1}{2} \varepsilon_{i j k} P^{m j} \dot{P}^{m k}
$$

The question about the uniqueness of the condition (7) and the representation (8) remains open.

\section{Astronomical Implications}

Having fixed a relativistic reference system to be used to model the motion of the Earth-based sites relative to the Earth's center of mass, we could adopt any a priori assumptions on the internal structure of the Earth relative to that reference system. Usually, these assumptions are aimed to simplify the equations of motion. Observations should verify to what extent these assumptions reflect the reality. In this respect general relativity gives no further complications as compared to Newtonian physics. The assumptions can be of kinematical or dynamical nature. For example, the assumption of 
a Newtonian-like rigid-body velocity distribution within the Earth in the geocentric reference system

$$
\frac{d}{d t} x_{a}^{i}(t)=\varepsilon_{i j k} \omega^{j}(t) x_{a}^{k}(t)
$$

is closer to the reality than similar assumption in a relativistic barycentric reference system $\left(\tilde{t}, \tilde{x}^{i}\right)$

$$
\frac{d}{d \tilde{t}}\left(\tilde{x}_{a}^{i}(\tilde{t})-\tilde{x}_{E}^{i}(\tilde{t})\right)=\varepsilon_{i j k} \tilde{\omega}^{j}(\tilde{t})\left(\tilde{x}_{a}^{k}(\tilde{t})-\tilde{x}_{E}^{i}(\tilde{t})\right)
$$

where $\tilde{x}_{E}^{i}(\tilde{t})$ is the barycentric position of the Earth's center of mass (see Brumberg, 1995 for a discussion of (16)-(17)). On the other hand, we could adopt some dynamical assumptions. For example, we can suppose that the post-Newtonian tensor of inertia $C^{i j}(t)$ rotates rigidly

$$
\frac{d}{d t} C^{i j}(t)=\varepsilon_{i k a} \omega^{k}(t) C^{a j}(t)+\varepsilon_{j k a} \omega^{k}(t) C^{a i}(t) .
$$

Let us note that the three assumptions (16), (17) and (18) being quite different in general relativity are equivalent in Newtonian mechanics.

We have seen above that the post-Newtonian rotational equations of motion of the Earth with respect to the relativistic geocentric reference system can be represented formally similar to their Newtonian counterparts. This means that many of the Newtonian results concerning Earth rotation with respect to its center of mass are also true in the post-Newtonian approximation of general relativity. Let us discuss some aspects of the rotational equations of motion specific to general relativity.

\subsection{POST-NEWTONIAN TERMS IN THE TENSOR OF INERTIA $C^{I J}$}

According to the definition (10), the post-Newtonian tensor of inertia $C^{i j}$ has several explicit relativistic contributions. Assuming the Earth to be spherically symmetric in order to estimate these relativistic terms, one gets (Klioner, 1995)

$$
\left\|C_{\mathrm{pN}}^{i j}-C_{\mathrm{N}}^{i j}\right\| \approx 1.9 \cdot 10^{-9} \delta^{i j} \mathcal{C}+\text { terms of order of } \sim 10^{-12} \mathcal{C},
$$

where $\mathcal{C}$ is the maximal principal moment of inertia of the Earth. The most precisely known parameter related directly to the tensor of inertia is probably dynamical ellipticity

$$
H=\frac{\mathcal{C}-\frac{1}{2}(\mathcal{A}+\mathcal{B})}{\mathcal{C}}
$$

where $\mathcal{A}, \mathcal{B}, \mathcal{C}$ are principal moments of inertia. Modern observations allow to measure the dynamical ellipticity with a precision of $\delta H / H \sim 10^{-5}$ $10^{-6}$. The post-Newtonian effects in the tensor of inertia $C^{i j}$ give

$$
\frac{\left|H_{\mathrm{pN}}-H_{\mathrm{N}}\right|}{H} \approx 1.9 \cdot 10^{-9}
$$


which is about 3 orders of magnitudes smaller. In the same time, in some recent papers devoted to the rigid-Earth nutation (see, e.g., Hartmann and Soffel, 1996), the dynamical ellipticity is fixed formally with 9-10 significant digits and, therefore, the last one or two digits are influenced by general relativity. Let us note that the definition (10) relates the post-Newtonian tensor of inertia $C^{i j}$ to matter variables (such as mass density, internal pressure, etc.). However, in astronomical practice these relations are probably not of much importance. The equations describing various observing quantities can be parameterized in such a way that they involve explicitly $C^{i j}$ as a whole. The value of $C^{i j}$ is derived directly from observations and its relation to the internal structure of the Earth is not always important.

\subsection{RELATION BETWEEN TENSOR OF INERTIA AND MASS QUADRUPOLE}

It can be shown that the basic Newtonian relation between Newtonian tensor of inertia $C_{\mathrm{N}}^{i j}$ and Newtonian mass quadrupole $M_{\mathrm{N}}^{i j}$

$$
C_{\mathrm{N}}^{i j}-\frac{1}{3} \delta^{i j} C_{\mathrm{N}}^{s s}=-M_{\mathrm{N}}^{i j}
$$

is no longer valid in general relativity

$$
C^{i j}-\frac{1}{3} \delta^{i j} C^{s s} \neq-M^{i j},
$$

where $M^{i j}$ is the post-Newtonian Blanchet-Damour quadrupole moment. The difference can be estimated as

$$
\left\|C^{i j}-\frac{1}{3} \delta^{i j} C^{s s}+M^{i j}\right\| \sim 10^{-12} \mathcal{C} .
$$

The principal observable parameter related to the mass quadrupole of the Earth is the second zonal harmonic $J_{2}$. Eq. (24) means in particular that in the usual definition of $J_{2}$

$$
J_{2}=\frac{\mathcal{C}^{\prime}-\frac{1}{2}\left(\mathcal{A}^{\prime}+\mathcal{B}^{\prime}\right)}{M R^{2}}
$$

one has to consider values $\mathcal{A}^{\prime}, \mathcal{B}^{\prime}, \mathcal{C}^{\prime}$ different from the principal moments of inertia used in the definition of dynamical ellipticity (20).

Again, we have to note that Eq. (23) is probably of no importance for astronomical practice since the values of $H$ and $J_{2}$ are derived independently and all the relativistic effects are masked by our poor knowledge of the Earth's internal structure. On the other hand, this effect can play an important role when modeling pulsar timing data (see Thorne and Gürsel, 1983).

\subsection{POST-NEWTONIAN EFFECTS IN TORQUE}

The post-Newtonian terms in the torque have a direct observable consequence as it has been computed by Voinov (1988) and Bizouard et al. 
(1992). The order of magnitude of this effect is $\sim 1$ microarcsecond in the Earth orientation parameters. The resulting expressions for this effect are different in the two mentioned papers and should be verified.

We have seen that if we use a physically adequate geocentric reference system relativistic effects in Earth rotation are quite small. Nevertheless, it is necessary to agree upon the definitions concerning the relativistic modeling of Earth rotation in order to avoid any possible discrepancy between the results coming from various kinds of geodynamical observations as well as any ambiguity in determination of the Earth orientation relative to remote quasars with an accuracy of the order of 1 microarcsecond.

Acknowledgements. This work was supported in part by a post-doctoral fellowship of the Alexander von Humboldt Foundation. The author is thankful to Prof. M. Soffel for numerous fruitful discussions.

\section{References}

Bizouard, C., Schastok, J., Soffel, M.H., and Souchay, J.: 1992, "Étude de la rotation de la Terre dans le cadre de la relativité générale: première approche", in: Journées 1992 (N. Capitaine, ed.), Obs. de Paris, 76-84.

Brumberg, V.A.: 1972, Relativistic Celestial Mechanics, Nauka, Moscow (in Russian).

Brumberg, V.A. and Kopejkin, S.M.: 1989, "Relativistic theory of celestial reference frames", in: Reference Frames (J. Kovalevsky, I.I. Mueller, B. Kolaczek, eds), Kluwer, Dordrecht, p.115.

Brumberg, V.A.: 1995, "General relativistic description of Earth's rotation in different reference systems" J. Geodyn. 20, 181-197.

Damour, T., Soffel, M., and Xu, C.: 1993, "General relativistic celestial mechanics III. Rotation equations of motion", Phys. Rev. D 47, 3124-3135.

Dixon, W.G.: 1979, "Extended bodies in general relativity: their description and motion", in: Isolated Gravitating Systems in General Relativity (J. Ehlers ed.), North-Holland, Amsterdam, 156-219.

Fock, V.A.: 1959, Theory of Space, Time and Gravitation, Pergamon, Oxford.

Hartmann, T. and Soffel, M.: 1996, "The nutation of a rigid Earth model: A complete new series from a high precision tidal potential development", Celest. Mech. \& Dyn. Astron., in press.

Klioner, S.A.: 1996, "Angular velocity of rotation of extended bodies in general relativity", in: Dynamics, Ephemerides and Astrometry of the Solar System, IAU Symposium 172 (S. Ferraz-Mello, B. Morando, J.E. Arlot, eds), Kluwer, Dordrecht, 309-320.

Klioner, S.A.: 1995, "Relativistic effects in orientation of astronomical reference frames", in: Earth Rotation, Reference Systems in Geodynamics and Solar System, Journées 1995 (N. Capitaine, B. Kolaczek, S. Debarbat eds), Warsaw, 175-178.

Moritz, H. and Mueller, I.I.: 1987, Earth Rotation: Theory and Observation, Ungar, New York.

Soffel, M.: 1994, "The problem of rotational motion and rigid bodies in the postNewtonian framework", unpublished notes.

Thorne, K.S. and Gürsel, Y.: 1983, "The free precession of slowly rotating neutron stars: rigid-body motion in general relativity", Mon. Not. R. Astron. Soc. 205, 809-817.

Voinov, A.V.: 1988, "Motion and rotation of celestial bodies in the post-Newtonian approximation", Celest. Mech. 41, 293-307. 